\title{
What advances have been made in immune-therapy for renal cell carcinoma?
}
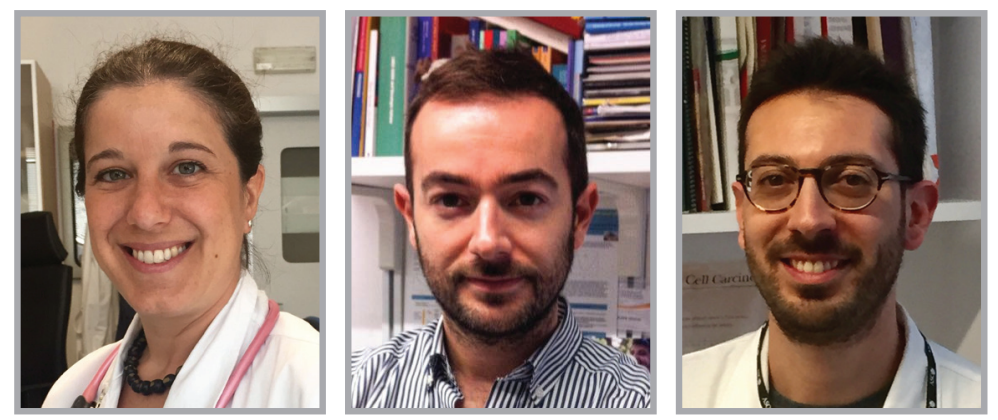

Elena Verzoni*, ${ }^{* 1}$, Paolo Grassi ${ }^{1} \&$ Raffaele Ratta ${ }^{1}$

First draft submitted: 16 January 2017; Accepted for publication: 27 January 2017; Published online: 10 February 2017

Renal cell carcinoma (RCC) is characterized by high immunogenicity, with a remarkable infiltration of immune cells, especially $\mathrm{T}$ cells, natural killer cells, dendritic cells and macrophages [1]. Before the advent of targeted therapy, cytokines including IL-2 and IFN- $\alpha$ were the standard of care in metastatic RCC (mRCC) treatment. Coppin et al. showed that IFN- $\alpha$ was associated with a small survival benefit (3.8 months) [2] . A retrospective analysis of seven Phase II trials testing high-dose IL-2 (HD IL-2) reported more durable responses in selected patients, with an objective response rate (ORR) of 14\% including $5 \%$ of complete responses [3]. However, most responses were partial and treatment was associated with severe side effects, such as hypotension, capillary leak syndrome, fever, edema, renal failure, dermatologic rashes and neurotoxicity. Different trials with new tyrosine kinase inhibitors (TKIs) had IFN- $\alpha$ as comparator but also the association with IL-2 was evaluated. The randomized, Phase II trial ROSORC trial, comparing sorafenib plus IL-2 versus sorafenib alone as firstline treatment of mRCC demonstrated a high percentage of partial responses (27\%) and stabilization of disease (53\%) in the combination arm but failed to demonstrate differences in progressionfree survival (PFS) [4]. More recently, McDermott et al. in the 'SELECT' trial enrolled 120 patients treated with HD IL-2 with a PFS of 4.4 months and an RR of $25 \%$ [5]. The aim of the study was to validate proposed predictive markers of response to HD IL-2. However, this model failed to identify selection criteria, since remissions and prolonged survival was achieved in both 'good' and 'poorrisk' patients, suggesting the need of other predictive markers.

In recent years, new agents targeting either the VEGFRs, such as sunitinib, sorafenib, pazopanib, axitinib and bevacizumab, and the mammalian target of rapamycin pathways (temsirolimus and everolimus) have changed the treatment

\section{KEYWORDS}

- immune-checkpoint inhibitors

- immunotherapy • renal cell carcinoma

“Renal cell carcinoma is characterized by high

immunogenicity, with a remarkable infiltration of immune cells, especially T cells, natural killer cells, dendritic cells and macrophages.” 
“Immune-checkpoint inhibitors have significantly improved survival of metastatic renal cell carcinoma patients and contributed to change our point of view on the end points for future clinical trials that should rather consider overall survival as primary end point." scenario of mRCC, improving survival rates and replacing the 'old' immunotherapy.

Over the last decade, significant improvements in the understanding of escape mechanisms to antitumor response have been made. Immune checkpoint inhibitors (ICIs) have produced durable response rates and survival improvement in different solid tumors and represent a major focus of research. Monoclonal antibodies (mAbs) directed against anti-PD-1 and anti-PD-L1 as well as against CTLA- 4 are currently being developed in clinical trials in most of the genitourinary malignancies. Two PD-1 inhibitors, nivolumab and pembrolizumab, as well as two PD-L1 inhibitors, atezolizumab and avelumab are being tested in RCC [6,7].

Pembrolizumab (MK-3475), a highly selective humanized IgG4 mAb targeting the PD-1 receptor, is under investigation in the neoadjuvant setting in patients with RCC (NCT02212730), as well as in combination with pazopanib (NCT02014636) and axitinib (NCT02133742) in the first-line setting. A Phase Ia study has investigated the efficacy and safety of atezolizumab, a $\mathrm{mAb}$ that targets PD-L1 in $63 \mathrm{mRCC}$ patients showing a median PFS of 5.6 months and a median OS of 28.9 months. The ORR was 15\% (18 and $9 \%$ in patients with $>1 \%$ and $<1 \%$ PD-L1 expression, respectively) [8]. A Phase $1 \mathrm{~b}$, open-label trial (NCT02493751) has investigated the maximum tolerated dose and selected the recommended Phase II dose of avelumab (MSB0010718C), a fully human anti-PD-L1 IgG1 mAb in combination with axitinib in patients with treatmentnaive mRCC. Among these agents, nivolumab is the only $\mathrm{mAb}$ approved by the US FDA and the EMA for the treatment of mRCC.

Nivolumab (BMS-936558), has been evaluated in Phase I and II trials of previously treated mRCC patients $[9,10]$ and on the basis of the promising results of these trials, it was compared with everolimus in the same setting in the randomized, Phase III CheckMate-025 study [11].

Median overall survival (OS), which was the primary end point, was 25 months in patients treated with nivolumab and 19.6 months in patients treated with everolimus. Nivolumab has also shown an increased ORR (25 vs 5\%). Patients treated with nivolumab had a median PFS of 4.6 versus 4.4 months in the everolimus arm. An ad hoc sensitivity analysis of PFS was performed in patients who did not progress or die at 6 months, showing that PFS in patients treated with nivolumab was 15.6 versus 11.7 months of those treated with everolimus. The most common side effects observed were fatigue, nausea, pruritus and diarrhea, but nivolumab had fewer grade 3-4 events than everolimus.

The subgroup analysis presented at the 2016 Genitourinary Cancers Symposium has confirmed the advantage of nivolumab in all subgroups analyzed pointing out an advantage in poor-risk patients according to the Memorial Sloan-Kettering Cancer Center prognostic score [12]. Benefit in OS was seen regardless of levels of PD-L1 expression.

Combination strategies with both ICIs and TKIs would certainly change our approach to the treatment of RCC. Nivolumab has shown promising results in combination with ipilimumab in a Phase I trial (CheckMate 016) presented at the 2014 American Society of Clinical Oncology (ASCO) annual meeting [13]. Ipilimumab is a mAb that inhibits CTLA-4, allowing cytotoxic $\mathrm{T}$ lymphocytes to recognize tumor cells previously perceived as normal. As a result of the acceptable safety profile and the encouraging efficacy, a Phase III trial is evaluating this combination in first line versus sunitinib (CheckMate 214, NCT02231749).

Nivolumab has also been associated with sunitinib or pazopanib. The rationale of the combination of ICIs with TKIs is that these agents are able to modulate the immune environment by decreasing the number of circulating regulatory T cells and myeloid-derived suppressor cells [14]. In a Phase I trial, interesting results in terms of ORR were seen $(52 \%$ in the sunitinib arm and $45 \%$ in the pazopanib arm), but the association was not manageable and pazopanib combination arm was even closed due to liver toxicity [15]. The high-toxicity profile underlines the difficulty in the development of such combinations; for these reasons the results of trials evaluating combination of immunotherapy with new generation TKIs (such as axitinib) are awaited.

Due to the promising results of ICIs in mRCC, it appears reasonable to evaluate the role of immunotherapy in the adjuvant setting. Various randomized trials of adjuvant therapy including vaccines, IFN- $\alpha$, HD IL-2 or a combination of cytokines failed to demonstrate survival benefit in comparison with observation [16]. To date, the role of ICIs has not been investigated in the adjuvant setting; however, some evidences suggest that resection of renal primary tumors may cause a decrease of PD-1 and PD-L1 expression, thus reducing the potential target for 
these agents [17,18]. By the way, several studies will evaluate the use of ICIs in either high-risk resected and oligo-mRCC patients undergoing metastasectomy without residual disease.

Despite these considerable advances, several questions remain to be answered.

First of all, the identification of possible predictive biomarkers of response as well as of a molecular profiling, that might guide treatment choice in every patient and predict response or resistance to ICIs, to avoid toxicity, maximize outcomes and reduce costs. Some genes involved in RCC pathogenesis include the DNA mismatch repair system ( $M L H 1$ and $M S H 2)$, PBRM1 and BAP1, and their loss can lead to an increased mutational load that may correlates with sensitivity to ICIs. Some studies have shown that inactivating mutations of PBMR1 and BAP1 are frequently associated with increased PD-L1 expression on RCC cells and concomitant PD-1 expression on tumor-infiltrating lymphocytes, suggesting that patients bearing this molecular profile could be good candidates for immunotherapy. This correlation needs to be prospectively validated in a clinical setting [19].

Second, the kinetics of response to ICIs is quite heterogeneous and might differ from those of TKIs. Objective responses can be observed from few weeks to several months after the beginning of therapy and apparent early disease progression may precede response or stable disease. For this reason, it is still unclear about the right timing required to assess treatment response. Sustained reductions in tumor burden or stabilization in the size of target lesions have been seen in patients who continued treatment with nivolumab beyond progression [20]. To this purpose, liquid biopsies or immunoprofiling may possibly help to distinguish between real progressive disease and pseudo-progression. In addition, these new agents have broaden the horizons of RCC treatment but we still need to understand the most effective setting for their use as well as the best sequencing in clinical practice.

ICIs have significantly improved survival of mRCC patients and contributed to change our point of view on the end points for future clinical trials that should rather consider OS as primary end point.

\section{Financial \& competing interests disclosure}

E Verzoni reports receiving fees for serving on advisory boards from Pfizer, Janssen and Novartis. The authors have no other relevant affiliations or financial involvement with any organization or entity with a financial interest in or financial conflict with the subject matter or materials discussed in the manuscript apart from those disclosed.

No writing assistance was utilized in the production of this manuscript.

\section{References}

1 Noessner E, Brech D, Mendler AN, Masouris I,Schlenker R, Prinz PU. Intratumoral alterations of dendritic-cell differentiation and CD8 ${ }^{+} \mathrm{T}$-cell anergy are immune escape mechanisms of clear cell renal cell carcinoma. Oncoimmunology 1(8), 1451-1453 (2012).

2 Coppin C, Porzsolt F, Awa A et al. Immunotherapy for advanced renal cell cancer. Cochrane Database Syst. Rev. 25 (1), CD001425 (2005).

3 Fyfe G, Fisher R, Rosenberg S et al. Results of treatment of 255 patients with metastatic renal cell carcinoma who received high-dose recombinant interleukin-2 therapy. J. Clin. Oncol. 13(3), 688-696 (1995).

4 Procopio G, Verzoni E, Bracarda S et al. Sorafenib with interleukin-2 vs sorafenib alone in metastatic renal cell carcinoma: the ROSORC trial. Br. J. Cancer 104(8), 1256-1261 (2011).

5 McDermott DF, Cheng SC, Signoretti S et al. The high-dose aldesleukin select trial: a trial to prospectively validate predictive models of response to treatment in patients with metastatic renal cell carcinoma. Clin. Cancer Res. 21, 561-568 (2015).

6 Ratta R, Zappasodi R, Raggi D et al. Immunotherapy advances in uro-genital malignancies. Crit. Rev. Oncol. Hematol. 105, 52-64 (2016).

7 Viteri S, González-Cao M, Barrón F, Riso A, Rosell R. Results of clinical trials with anti-programmed death 1 /programmed death ligand 1 inhibitors in lung cancer. Transl. Lung Cancer Res. 4(6), 756-762 (2015).

8 McDermott DF, Sosman JA, Sznol M et al. Atezolizumab, an anti-programmed death-ligand 1 antibody, in metastatic renal cell carcinoma: long-term safety, clinical activity, and immune correlates from a Phase Ia study. J. Clin. Oncol. 34(8), 833-842 (2016).

9 Topalian S, Hodi F, Brahmer J et al. Safety, activity, and immune correlates of anti-PD-1 antibody in cancer. N. Engl. J. Med. 366, 2443-2454 (2012).
10 Motzer RJ, Rini BI, McDermott DF et al. Nivolumab for metastatic renal cell carcinoma: results of a randomized Phase II trial. J. Clin. Oncol. 33(13), 1430-1437 (2015).

11 Motzer R, Escudier B, McDermott D et al. Nivolumab versus everolimus in advanced renal-cell carcinoma. N. Engl. J. Med. 373, 1803-1813 (2015).

12 Motzer R, Sharma P, McDermott D et al. CheckMate 025 Phase III trial: outcomes by key baseline factors and prior therapy for nivolumab (NIVO) versus everolimus (EVE) in advanced renal cell carcinoma (RCC). J. Clin. Oncol. 34(Suppl. 2S), Abstract 498 (2016).

13 Hammers H, Plimack E, Infante J et al. Phase I study of nivolumab in combination with ipilimumab inmetastatic renal cell carcinoma (mRCC). J. Clin. Oncol. 32, Abstract 4504 (2014).

14 Ko JS, Rayman P, Ireland J et al. Direct and differential suppression of myeloid-derived suppressor cell subsets by sunitinib is 
EDITORIAL Verzoni, Grassi \& Ratta

compartimentally constrained. Cancer Res. 70, 3526-3536 (2010).

15 Amin A, Plimack E, Infante J et al. Nivolumab (anti-PD-1; BMS-936558, ONO-4538) in combination with sunitinib or pazopanib in patients (pts) with metastatic renal cell carcinoma (mRCC). J. Clin. Oncol. 32(Suppl. 15), 5010 (2014).

16 Smaldone MC, Fung C, Uzzo RG, Haas NB. Adjuvant and neoadjuvant therapies in high-risk renal cell carcinoma. Hematol. Oncol. Clin. North Am. 25(4), 765-791 (2011).
17 MacFarlane AW 4th, Jillab M, Plimack ER et al. PD-1 expression on peripheral blood cells increases with stage in renal cell carcinoma patients and is rapidly reduced after surgical tumor resection. Cancer Immunol. Res. 2(4), 320-331 (2014).

18 Jilaveanu LB, Shuch B, Zito CR et al. PD-L1 expression in clear cell renal cell carcinoma: an analysis of nephrectomy and sites of metastases. J. Cancer 5(3), 166-172 (2014).

19 Joseph RW, Kapur P, Serie DJ et al. Loss of BAP1 protein expression is an independent marker of poor prognosis in patients with lowrisk clear cell renal cell carcinoma. Cancer 120(7), 1059-1067 (2014).

20 George S, Motzer RJ, Hammers HJ et al. Safety and efficacy of nivolumab in patients with metastatic renal cell carcinoma treated beyond progression: a subgroup analysis of a randomized clinical trial. JAMA Oncol. 2(9), 1179-1186 (2016). 